\title{
Photoemission spectrum in paramagnetic FeO under pressure: Towards an $a b$ initio description
}

\author{
S. Di Sabatino $\odot,{ }^{1,2}$ J. Koskelo $\odot,{ }^{1}$ J. A. Berger $\odot,^{2}$ and P. Romaniello $\odot^{1, *}$ \\ ${ }^{1}$ Laboratoire de Physique Théorique, Université de Toulouse, CNRS, UPS and ETSF, France \\ ${ }^{2}$ Laboratoire de Chimie et Physique Quantiques, Université de Toulouse, CNRS, UPS and ETSF, France
}

(Received 25 February 2020; revised 22 December 2020; accepted 26 January 2021; published 22 February 2021)

In this paper we provide an exhaustive study of the photoemission spectrum of paramagnetic $\mathrm{FeO}$ under pressure using a refined version of our recently derived many-body effective energy theory (MEET). We show that, within a nonmagnetic description of the paramagnetic phase, the MEET gives an overall good description of the photoemission spectrum at ambient pressure as well as the changes it undergoes by increasing pressure. In particular at ambient pressure the band gap opens between the mixed Fe $t_{2 g}$ and $\mathrm{O} 2 p$ states and the Fe $4 s$ states and, moreover, a $d$ - $d$ gap opens, which is compatible with a high-spin configuration (hence nonzero local magnetic moments as observed in experiment), whereas with decreasing pressure the band gap tends to close, $t_{2 g}$ states tend to become fully occupied, and $e_{g}$ states tend to become fully unoccupied, which is compatible with a low-spin configuration (hence a collapse of the magnetic moments as observed in experiment). This is a remarkable result, since, within a nonmagnetic description of the paramagnetic phase, the MEET is capable to correctly describe the photoemission spectrum and the spin configuration at ambient as well as high pressure. For comparison we report the band-gap values obtained using density-functional theory with a hybrid functional containing screened exchange (HSE06) and a variant of the $G W$ method (self-consistent COHSEX), which are reliable for the description of the antiferromagnetic phase. Both methods open a gap at ambient pressure, although, by construction, they give a low-spin configuration; increasing pressure they correctly describe the band-gap closing. We also report the photoemission spectrum of the metallic phase obtained with one-shot fully dynamical $G W$ on top of the local-density approximation, which gives a spectrum very similar to dynamical mean-field theory results from literature.

DOI: 10.1103/PhysRevResearch.3.013172

\section{INTRODUCTION}

$\mathrm{FeO}$ is a basic oxide component of the Earth's interior [1] and has a rather complex pressure-temperature phase diagram [2-6]. It was found experimentally that, under ambient conditions, it is a paramagnetic insulator with a rocksalt B1 crystal structure and it undergoes a phase transition into rhombohedrally distorted B1 structure above $16 \mathrm{GPa}$ [7]. At high temperature and pressure an insulator-to-metal transition is observed with a collapse of the local magnetic moment [2,4]. A similar magnetic collapse at high pressure is also measured in other similar transition-metal (TM) oxides such as $\mathrm{MnO}$, $\mathrm{CoO}$, and $\mathrm{NiO}[5,6,8,9]$.

Various detailed theoretical studies of the electronic structure and phase stability of these transition-metal oxides, in particular in the paramagnetic phase (PM), which employ dynamical mean-field theory (DMFT) combined with ab initio band-structure methods, are present in the literature, which interpret these systems as Mott insulators [10-14]. However, Trimarchi et al. [15] have shown that a correct description of

\footnotetext{
*pina.romaniello@irsamc.ups-tlse.fr

Published by the American Physical Society under the terms of the Creative Commons Attribution 4.0 International license. Further distribution of this work must maintain attribution to the author(s) and the published article's title, journal citation, and DOI.
}

these systems can also be obtained using only band-structure theories provided that one models the spin-disordered PM phase using a larger supercell where each TM site can have different spin direction and different local bonding geometry, but still with a zero total spin. Within this so-called polymorphous band-structure description the band-gap opening is not driven by strong correlation as in DMFT.

In this paper we investigate the electronic structure of paramagnetic $\mathrm{FeO}$ both at ambient and at high pressure within an $a b$ initio framework. We adopt the DMFT standpoint, in which the existence of local magnetic moments is a consequence of the electron localization and not an essential part of the gap opening mechanism itself. We therefore model the system as nonmagnetic (spin unpolarized). Paramagnetic $\mathrm{FeO}$ is a special case because within a nonmagnetic modeling of the PM phase Hartree-Fock (HF) theory is enough to open a gap in this system, unlike in similar systems, such as $\mathrm{MnO}$, $\mathrm{CoO}$, and $\mathrm{NiO}$ [16]. This occurs because nonlocal exchange well separates in energy the transition-metal $d$ states with $t_{2 g}$ symmetry from those with $e_{g}$ symmetry. Since Fe has six $d$ electrons, they fully occupy the $t_{2 g}$, opening a gap. In other similar transition-metal oxides, this does not happen since the transition metal has less than or more than six electrons, and therefore they partially occupy the $t_{2 g}$ or $e_{g}$ bands, leading to a metal. This happens because in the nonmagnetic case static mean-field theories can only open a gap if there is an even number of electrons, in which case a band insulator 
forms, or if there is a spontaneously broken spin and/or translational symmetry (e.g., magnetic order). Of course within such a nonmagnetic description of the paramagnetic phase local magnetic moments are zero by construction. Mean-field methods are more appropriate in the high-pressure regime, where the band gap closes and the system is in a low-spin configuration. We show, for example, that generalized KohnSham theory within the HSE06 functional [17] can describe the band-gap closing by increasing pressure. Instead, using a refined version of our recently derived many-body effective energy theory (MEET), one can get an overall good description of the photoemission spectrum (PES) and spin configuration at ambient and high pressure, yet remaining in a nonmagnetic description of the PM phase. This paper is organized as follows: in Sec. II we describe the MEET; in Sec. III we summarize the computational details of the performed calculations. Results of the photoemission spectrum at ambient as well as high pressure are presented and discussed in Sec. IV. Summary and conclusions are drawn in Sec. V.

\section{THEORETICAL BACKGROUND: THE MEET}

Within the MEET the time-ordered one-body Green's function $G(\omega)$ at zero temperature, which gives the spectral function as $A(\omega)=1 / \pi|\operatorname{Im} G(\omega)|$, is split into removal $(R)$ and addition $(A)$ parts as $G(\omega)=G^{R}(\omega)+G^{A}(\omega)$. The spectral function is the key quantity which is related to photoemission spectra. The diagonal matrix elements of $G^{R / A}(\omega)$ can be written in terms of an effective energy $\delta_{i}^{R / A}(\omega)$ as

$$
\begin{aligned}
G_{i i}^{R}(\omega) & =\frac{n_{i}}{\omega-\delta_{i}^{R}(\omega)-\mathrm{i} \eta}, \\
G_{i i}^{A}(\omega) & =\frac{1-n_{i}}{\omega-\delta_{i}^{A}(\omega)+\mathrm{i} \eta},
\end{aligned}
$$

where we use the basis of natural orbitals, i.e., the orbitals which diagonalize the one-body reduced density matrix (1RDM), with $n_{i}$ the occupation number of state $i$. Since the spectral function is expressed as

$$
A_{i}(\omega)=n_{i} \delta\left(\omega-\delta_{i}^{R}(\omega)\right)+\left(1-n_{i}\right) \delta\left(\omega-\delta_{i}^{A}(\omega)\right)
$$

fractional occupation numbers could lead to a gap opening in the degenerate $d$ states. We will come back to this point in Sec. IV A. The effective energy $\delta_{i}^{R / A}(\omega)$ can be written as an expansion in terms of reduced density matrices. The expressions truncated at the level of the 1-RDM and two-body reduced density matrix (2-RDM) read

$$
\begin{aligned}
\delta_{i}^{R,(1)} & =h_{i i}+\sum_{j} V_{i j i j} n_{j}+\frac{1}{n_{i}} \sum_{j k l} V_{i j k l} \Gamma_{\mathrm{xc}, k l j i}^{(2)}, \\
\delta_{i}^{A,(1)}= & h_{i i}+\sum_{j} V_{i j i j} n_{j} \\
& -\frac{1}{\left(1-n_{i}\right)}\left[\sum_{j} V_{i j j i} n_{j}+\sum_{j k l} V_{i j k l} \Gamma_{\mathrm{xc}, k l j i}^{(2)}\right],
\end{aligned}
$$

where $h_{i j}=\int d \mathbf{x} \phi_{i}^{*}(\mathbf{x}) h(\mathbf{r}) \phi_{j}(\mathbf{x})$ are the matrix elements of the one-particle noninteracting Hamiltonian $h(\mathbf{r})=-\nabla^{2} / 2+$ $v_{\text {ext }}(\mathbf{r})$ and $V_{i j k l}=\int d \mathbf{x} d \mathbf{x}^{\prime} \phi_{i}^{*}(\mathbf{x}) \phi_{j}^{*}\left(\mathbf{x}^{\prime}\right) v_{c}\left(\mathbf{r}, \mathbf{r}^{\prime}\right) \phi_{k}(\mathbf{x}) \phi_{l}\left(\mathbf{x}^{\prime}\right)$ are the matrix elements of the Coulomb interaction $v_{c}$.
Here we approximate the exchange-correlation contribution to the 2-RDM in terms of the power functional $\Gamma_{\mathrm{xc}, k l j i}^{(2)}=$ $-n_{i}^{\alpha} n_{j}^{\alpha} \delta_{i l} \delta_{j k}$, with $0.5 \leqslant \alpha \leqslant 1$ [19]. Moreover the natural orbitals and occupation numbers are obtained within reduceddensity matrix functional theory [18], where again the power functional [19] is employed to approximate the 2-RDM in the total-energy minimization. The approximation to the 2-RDM is extremely important, because the performance of the MEET heavily relies on it. The power functional has been tested on various extended systems [19-21], but the results on the occupation numbers are scarce [22]. In the MEET one needs accurate occupation numbers and this is not clearly guaranteed using the power functional. At high pressure, indeed, we have to vary $\alpha$ in order to have a low-spin configuration. This is discussed in the next section.

The MEET, at the level of $\delta^{R / A,(1)}$, gives qualitatively good spectra in gapped materials, but hugely overestimates the band gap; moreover it opens a gap even in metals. More details can be found in Refs. [16,23]. This is mainly due to the cutoff of the MEET equations at the level of the 1- and 2RDMs. This can be understood by reformulating $\delta_{i}^{R / A}(\omega)$ in terms of moments $\mu_{n, i}^{R / A}=\sum_{k} B_{i i}^{k, R / A}\left(\epsilon_{k}^{R / A}\right)^{n} / \sum_{k} B_{i i}^{k, R / A}$ (with $n$ indicating the order of the moment and $B_{i i}^{k, R / A}$ the spectral weight of the energy $\epsilon_{k}^{R / A}$ ) of $G_{i i}^{R / A}(\omega)$, as reported in Ref. [16]. The approximation $\delta_{i}^{R / A,(1)}$ is equal to the first moment $\mu_{1, i}^{R / A}$, which is a weighted average of all of the poles of $G_{i i}^{R / A}(\omega)$. Let us suppose that the photoemission spectrum is composed of quasiparticle peaks with roughly 30\% [24] of the spectral weight transferred to the corresponding satellites for the weakly correlated states (i.e., states characterized by natural occupation numbers close to 1 or 0 ) and a larger percentage for the strongly correlated (states characterized by fractional natural occupation numbers) $d$ states. Let us also assume that there is a single dominant plasmon which generates the satellites. The approximation $\delta_{i}^{R,(1)}\left(\delta_{i}^{A,(1)}\right)$ produces a removal (addition) peak which is redshifted (blueshifted) with respect to the exact quasiparticle; the shift is proportional to the spectral weight of the corresponding satellites. This explains (i) the overall good relative position of the spectral features in the removal and the addition parts of the MEET spectrum, with the $d$ states slightly redshifted (blueshifted) with respect to the rest of the removal (addition) part; and (ii) the overestimation of the band gap. An approximate estimation of the influence of the three-body reduced density matrix on the spectrum by screening the interaction in front of the 2-RDM in Eq. (4) indicates an improvement of the band gap [23]. We notice that also the approximations to the 2-RDM play a role in the final result. Work to understand the impact of the two approximations and how to include the effect of higher-order RDMs is currently in progress. Here we overcome the bandgap problem of the MEET using a pragmatic approach by aligning the weakly correlated MEET states (namely, O $2 p$ and $\mathrm{Fe} 4 s$ for the removal and the addition parts, respectively) to the local-density approximation (LDA) states. The rationale behind this procedure is the following.

(i) Removal and addition parts of the PES, separately, are overall well described by the MEET and a rigid shift of the two parts towards one another would suffice to reduce the fundamental gap. 


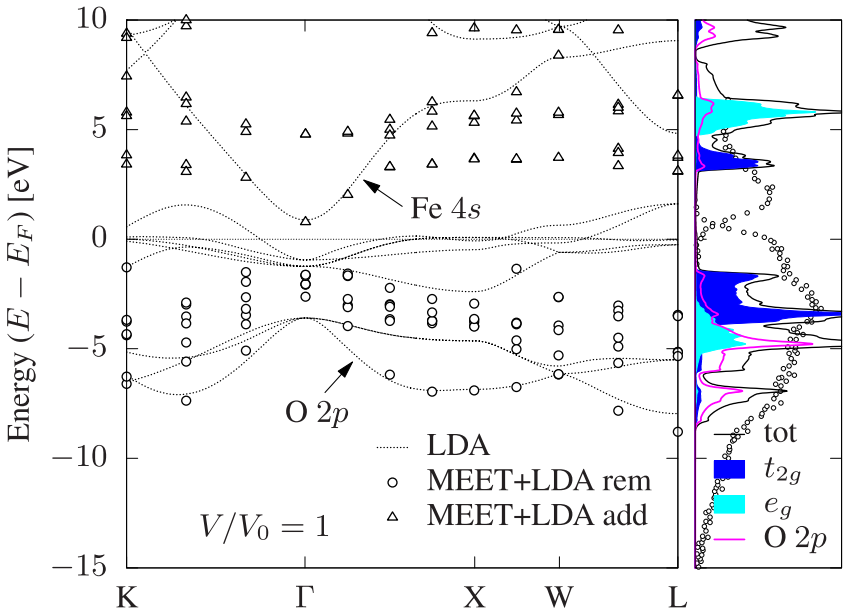

(a)

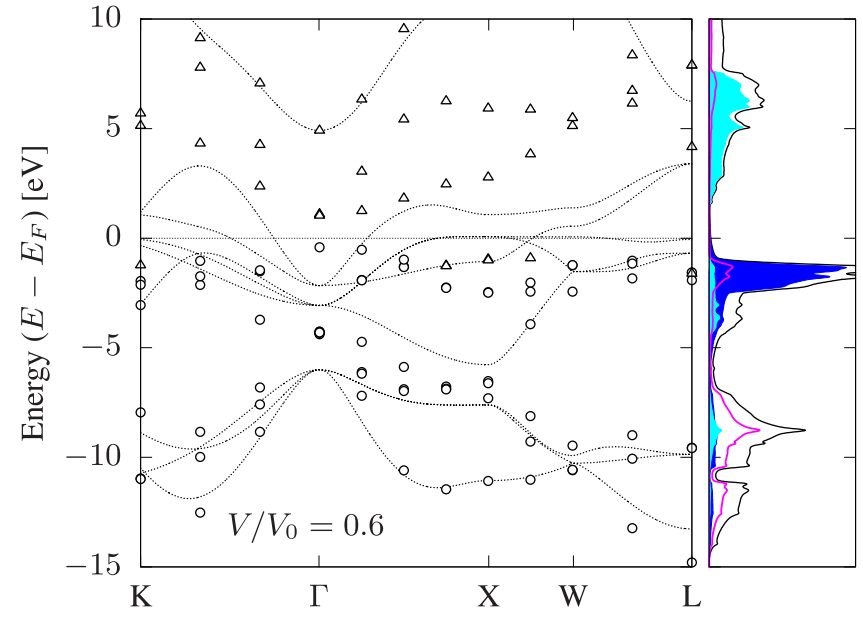

(b)

FIG. 1. MEET + LDA k-resolved and total spectral functions. Removal (circles) and addition energies (triangles) are reported along high-symmetry directions for relative volumes $V / V_{0}=1$ (a) and $V / V_{0}=0.6$ (b), where $V_{0}$ is the equilibrium volume at ambient pressure. We also report the LDA band structure (black dotted line). The contributions of the Fe $t_{2 g}$ and $e_{g}$ states and of the $\mathrm{O} 2 p$ states to the total spectral function are also reported. The experimental photoemission spectrum (small circles) is taken from Ref. [25].

(ii) In the spirit of DMFT the weakly correlated states are assumed to be well described by LDA.

The alignment yields the rigid shift we are seeking. In the following we will refer to this protocol as MEET + LDA.

\section{COMPUTATIONAL DETAILS}

The MEET calculations have been performed using a modified version of the open-source full-potential linearized augmented plane-wave code ELK [26], with practical details of the calculations following the scheme described in Ref. [19]. HSE06 and self-consistent Coulomb hole plus screened exchange (scCOHSEX) calculations have been performed using the Vienna Ab-Initio Simulation Package (VASP) [27-30] with the projector-augmented wave method [31,32]. For calculations of the $G_{0} W_{0}$ spectral function we used the ABINIT code $[33,34]$ employing plane waves and norm-conserving pseudopotentials.

We described the PM phase of $\mathrm{FeO}$ as nonmagnetic and we used a rocksalt structure with the experimental lattice constant, i.e., $4.33 \AA$ [35].

For the ELK calculations we used a $\Gamma$-centered $8 \times 8 \times 8 \mathbf{k}$ point grid. The muffin-tin (MT) radii for Fe and $\mathrm{O}$ atoms are 2.31 and 1.73 a.u., respectively. The MT radius times maximum $|\mathbf{G}+\mathbf{k}|$ vectors, $R^{\mathrm{MT}} \times \max |\mathbf{G}+\mathbf{k}|$, is 7.0 , while the maximum length of $|\mathbf{G}|$ for expanding the interstitial density and potential is 12.0 a.u. ${ }^{-1}$. We included eight empty bands.

In the $G_{0} W_{0}$ and scCOHSEX calculations we treated the dielectric screening at the level of the random-phase approximation, and included $\mathrm{Fe} 3 s, 3 p, 3 d$, and $4 s$ and $\mathrm{O} 2 s$ and $2 p$ as valence states. In the VASP calculations we included 200 bands for screening and self-energy, and expanded the pseudo wave functions and dielectric matrices in the basis of plane waves up to 700 and $800 \mathrm{eV}$, respectively. We used a $\Gamma$-centered $8 \times 8 \times 8 \mathbf{k}$-point grid.
In the ABINIT calculations we employed optimized normconserving Vanderbilt pseudopotentials [36,37], and expanded the ground-state wave functions up to $90 \mathrm{Ha}$. We used 100 bands for building the screening and self-energy. In the screening calculations we expanded the wave functions and screening matrix in plane waves up to 30 and $10 \mathrm{Ha}$, respectively. In the self-energy calculations we used plane waves up to $60 \mathrm{Ha}$ for both wave functions and Fock operator. We employed a $\Gamma$-centered $12 \times 12 \times 12 \mathbf{k}$-point grid. The frequency dependence of the self-energy was treated with the contour-deformation technique [38]. We sampled the imaginary axis with 25 frequencies, and real axis with 40 frequencies up to $20 \mathrm{eV}$.

\section{RESULTS: SPECTRAL FUNCTION}

\section{A. Many-body effective energy theory}

In Fig. 1 we report the spectral function of PM FeO at ambient pressure (left panel) and at high pressure (right panel) calculated using the MEET + LDA. The MEET removal and addition energies are aligned using the LDA bands corresponding to the weakly correlated $\mathrm{O} 2 p$ and Fe $4 s$ states, respectively.

At ambient pressure we use $\alpha=0.65$ for the power functional, as suggested in literature [19]. At high pressure we use $\alpha=0.70$. This value guarantees a decrease of the $e_{g}$ occupancy in favor of the $t_{2 g}$ occupancy, as illustrated in Fig. 2, and thus a transition to a low-spin configuration. More specifically this value of $\alpha$ is fixed by the $t_{2 g} / e_{g}$ occupation ratio at relative volume $V / V_{0}=0.6$ (with $V_{0}$ the equilibrium volume at ambient pressure) being approximately equal to the value deduced from Ref. [13] (see note [39]).

Unfortunately, there are very few experiments reporting the band gaps of $\mathrm{FeO}$ in the PM phase at ambient pressure, because of the difficulty to prepare pure FeO samples. We only found the value of $2.4 \mathrm{eV}$ from an optical absorption 

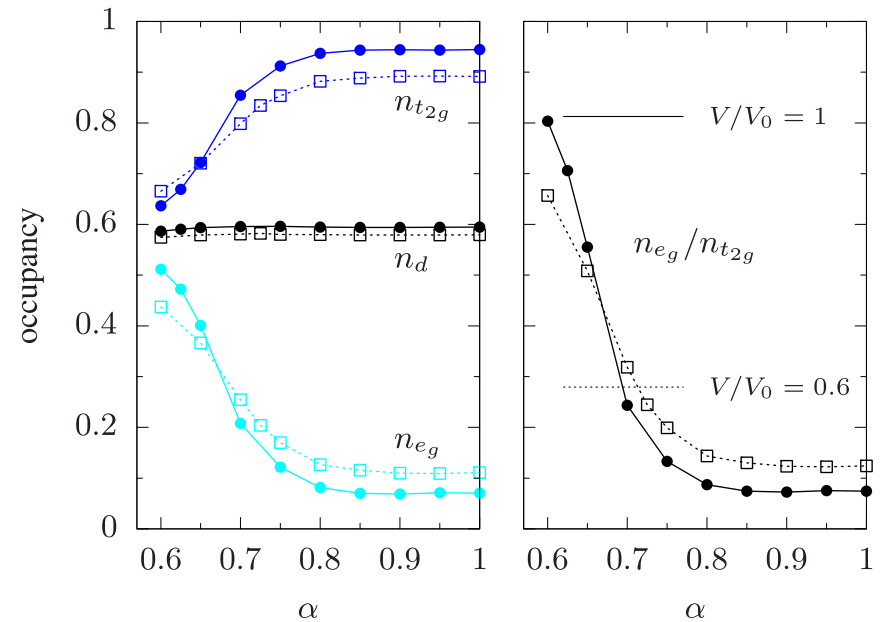

FIG. 2. Left panel: Occupancies of $t_{2 g}$ (blue) and $e_{g}$ (cyan) orbitals at relative volume $V / V_{0}=1$ (solid line and filled circles) and $V / V_{0}=0.6$ (dashed line and open squares) as a function of the power functional parameter $\alpha$. We report also the average $d$ occupancy (black). In the right panel the ratio between $e_{g}$ and $t_{2 g}$ occupancies is plotted. Values of the ratio $n_{e_{g}} / n_{t_{2 g}}$ are extracted from Ref. [13] and reported as horizontal lines.

measurement [40]. Overall the theoretical photoemission spectrum compares well with the experimental one [25]. The calculated band gap opens between the mixed Fe $t_{2 g}$ and $\mathrm{O}$ $2 p$ states and the Fe $4 s$ states with a value of about $2.1 \mathrm{eV}$. The $d$ - $d$ gap is instead about $4.4 \mathrm{eV}$. The MEET + LDA k-resolved spectral function compares rather well with DMFT results from Ref. [13] as one can see from Fig. 3.

The gap opening in the $d$-state manifold occurs because for values of $\alpha<1$ the occupation numbers, which enter the expression of the MEET spectral function [see Eq. (3)], are fractional and, hence, each of them gives rise to a removal energy and an addition energy. This is not the case for $\alpha=1$ (which is Hartree-Fock), since in this case the occupation numbers are either 1 or 0 , and therefore the $t_{2 g}$ states, which are fully occupied, will give rise only to removal energies, and the $e_{g}$ states, which are fully unoccupied, will give rise only to addition energies. The Fe $t_{2 g} / e_{g}$ orbital contributions to the spectral function reported in Fig. 1 are compatible with a high-spin configuration and, therefore, in line with the experimental observation of a local magnetic moment. This is a quite remarkable result, since, within a nonmagnetic description of the PM phase, the MEET + LDA is capable to give an overall good description of both the spectral function and the high-spin configuration.

In Fig. 4 we also report the spectral function of $\mathrm{FeO}$ at ambient pressure and high pressure (right panel) calculated using the MEET (i.e., without the alignment) with various values of the $\alpha$ parameter. We see that increasing $\alpha$, and hence decreasing the degree of correlation treated, tends to reduce the gap but also to deform the spectrum, in particular because the $t_{2 g}$ component in the addition part of the spectrum starts to migrate to lower energies while decreasing in intensity, as the occupation numbers tend towards 0 or 1 . As already anticipated, at $\alpha=1$ one gets the HF spectrum, which is compatible with a low-spin configuration. We also notice that HF opens a gap also at high pressure.

In the right panel of Fig. 1 we report the spectral function of $\mathrm{FeO}$ at high pressure (at relative volume $V / V_{0}=0.6$ ). At this reduced volume MEET + LDA results go in the right direction: the band gap closes, although the spectral weight around the Fermi energy is still too small (at least with respect to DMFT results, see Ref. [13]). The MEET + LDA $\mathbf{k}$-resolved spectral function compares well with DMFT results from Ref. [13], although the $d$ conduction bands are largely blueshifted with respect to DMFT results, as one can see from Fig. 3. We notice that to compare our results with DMFT we use the same lattice constant as in Ref. [13], namely, $4.42 \AA$ (8.36 a.u.), for the MEET + LDA results reported in Fig. 3.

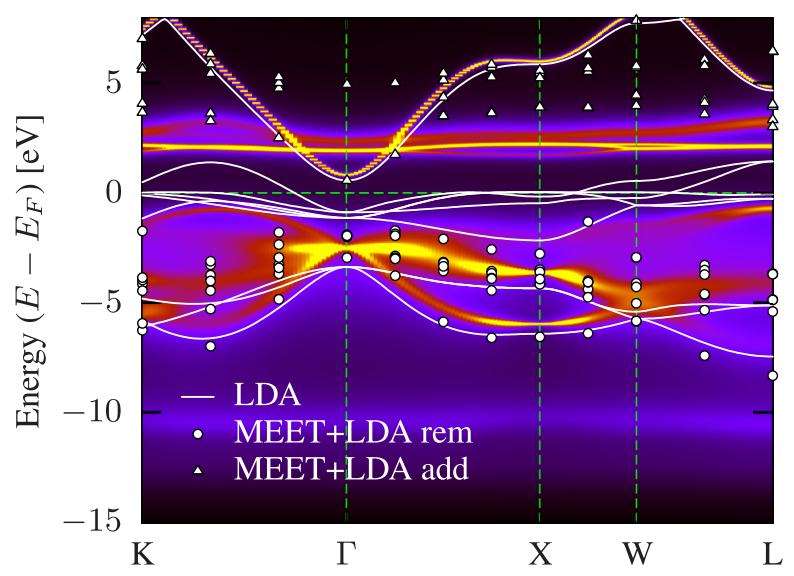

(a)

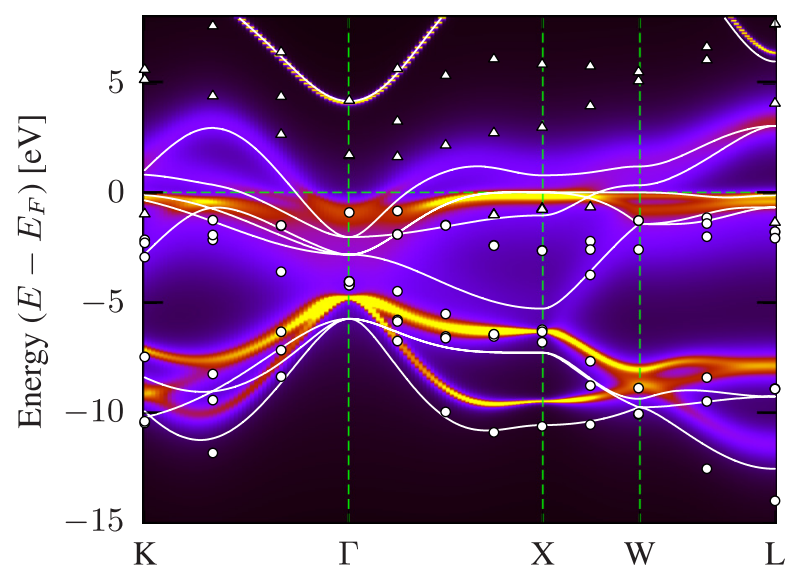

(b)

FIG. 3. k-resolved spectral function of FeO: MEET + LDA energies are reported along high-symmetry directions and compared with DMFT results from Ref. [13] for ambient (a) and high pressure (b). We also report LDA band structures (white solid line) which are used to align the MEET energies. Note that for a meaningful comparison we have used for MEET and LDA calculations the same lattice constant used in Ref. [13]. 


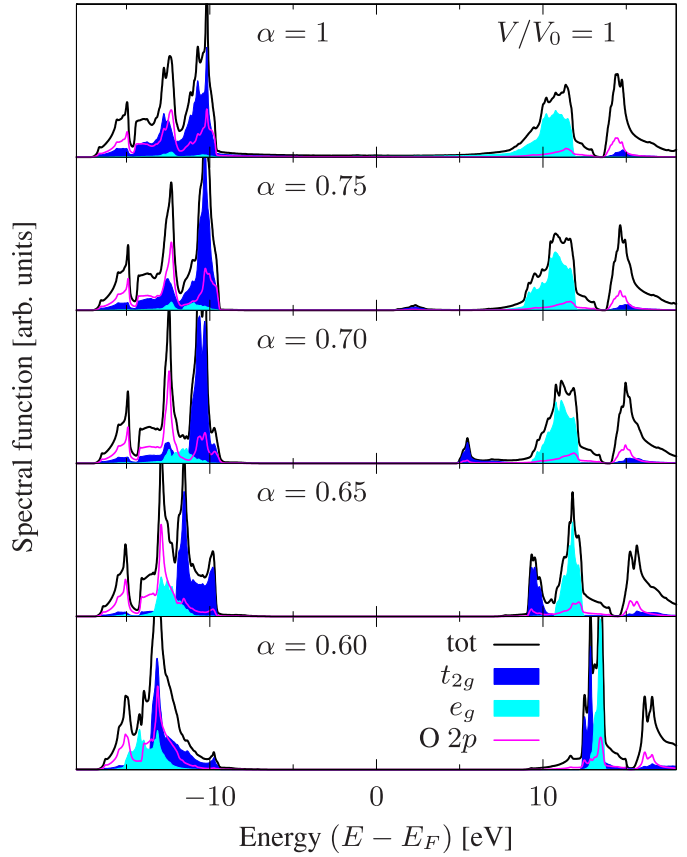

(a)

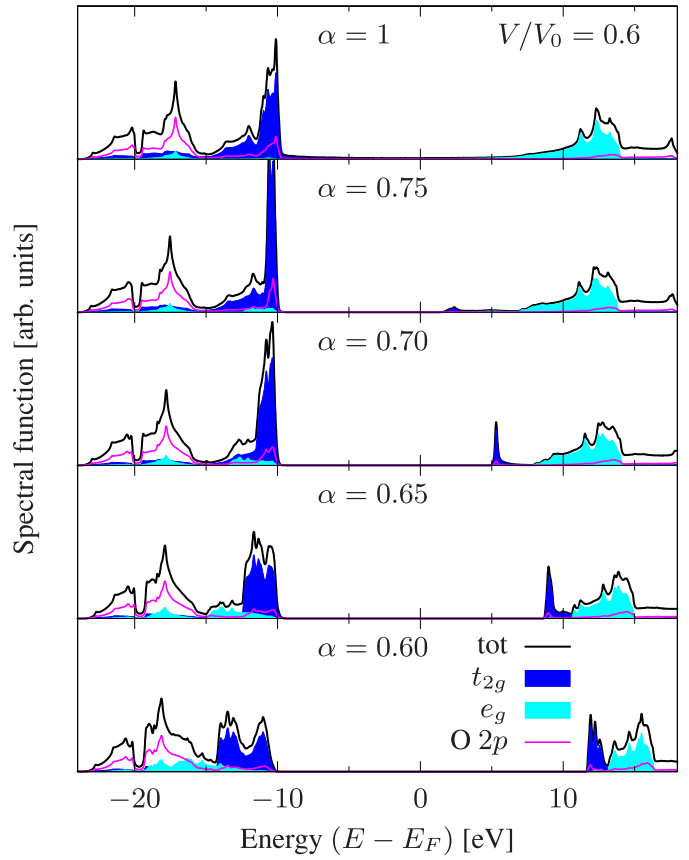

(b)

FIG. 4. Photoemission spectrum calculated using MEET (i.e., without the alignment with LDA) for various values of $\alpha$ for $V / V_{0}=1$ (a) and $V / V_{0}=0.6$ (b). The contributions of the Fe $t_{2 g}$ and $e_{g}$ states and of the $\mathrm{O} 2 p$ states to the total DOS are also reported.

\section{B. HSE06 and $G W$}

For comparison we also report our results obtained within the hybrid functional HSE06 and $G W$, which have been applied to transition-metal oxides in the antiferromagnetic phase at ambient pressure with relatively good results [41-43]. In Table I we report the band gap of $\mathrm{FeO}$ vs the relative volume $V / V_{0}$ calculated using HSE06 and scCOHSEX. COHSEX is a static variant of $G W$ [44], which allows for a computationally more feasible self-consistent solution; being static it reproduces only the quasiparticle peaks in the spectral function. While these methods give an incorrect picture at ambient pressure, as already discussed in the introduction, they are justified at high pressure, where the fundamental band gap closes and the system goes in a low-spin configuration. We observe that within both methods the system is a metal at $V / V_{0}=0.45$, with scCOHSEX closing the gap slightly faster (with respect to the volume decrease) than HSE06. In Fig. 5 we report the quasiparticle $(\mathrm{QP})$ band structure and DOS of the metallic phase (at $V / V_{0}=0.6$ ) calculated with one-shot $G W\left(G_{0} W_{0}\right)$ on top of LDA (using the linearized QP equation).

TABLE I. Fundamental band gap (eV) vs relative volume $V / V_{0}$. The experimental value at ambient pressure is $2.4 \mathrm{eV}$ from an optical absorption measurement [40].

\begin{tabular}{lcc}
\hline \hline$V / V_{0}$ & HSE06 & scCOHSEX \\
\hline 1 & 2.9 & 4.1 \\
0.75 & 2.2 & 3.0 \\
0.625 & 1.5 & 1.9 \\
0.50 & 0.4 & 0 \\
0.45 & 0 & 0 \\
\hline \hline
\end{tabular}

For comparison also the LDA band structure and DOS are reported. $G W$ induces a renormalization (although small) of the $d$ bands and lowers the $\mathrm{O} 2 p$ bands, with an increase of the gap with the $d$ states. The full (i.e., taking into account the full frequency dependence of the self-energy) $G_{0} W_{0}$ spectral function reported in Fig. 6 (upper panel) is in overall good agreement with DMFT results from literature, although the description of satellites could improve by introducing some form of self-consistency [45]. The shoulder at about $-7 \mathrm{eV}$ and the peak around $7 \mathrm{eV}$ largely come from $d$-band satellites as one can see in Fig. 6 (upper panel), where the QP and full $G_{0} W_{0}$ spectral functions are compared. These satellites are missing in the MEET within the current static approximation

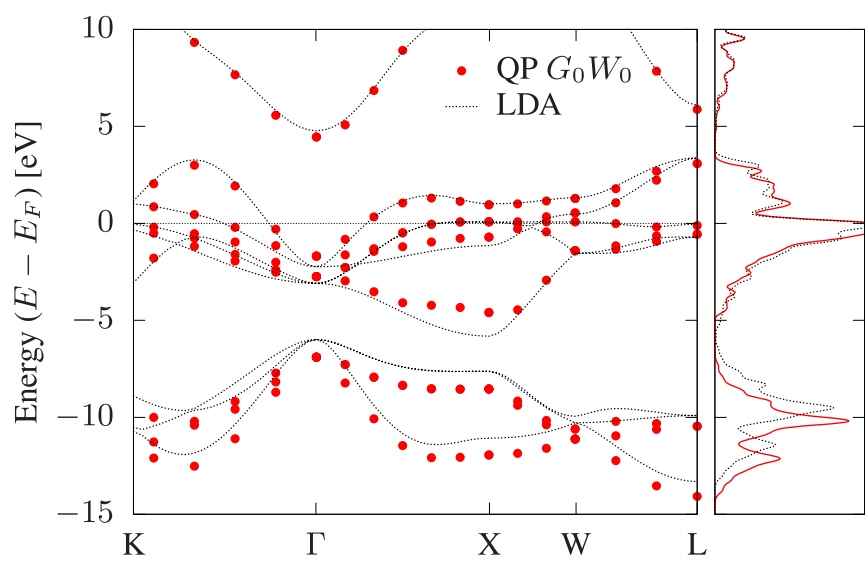

FIG. 5. Quasiparticle band structure (red dots) and spectral function (red solid line) within $G_{0} W_{0}$ at $V / V_{0}=0.6$. For comparison we report also the LDA band structure and DOS (dotted black line). 


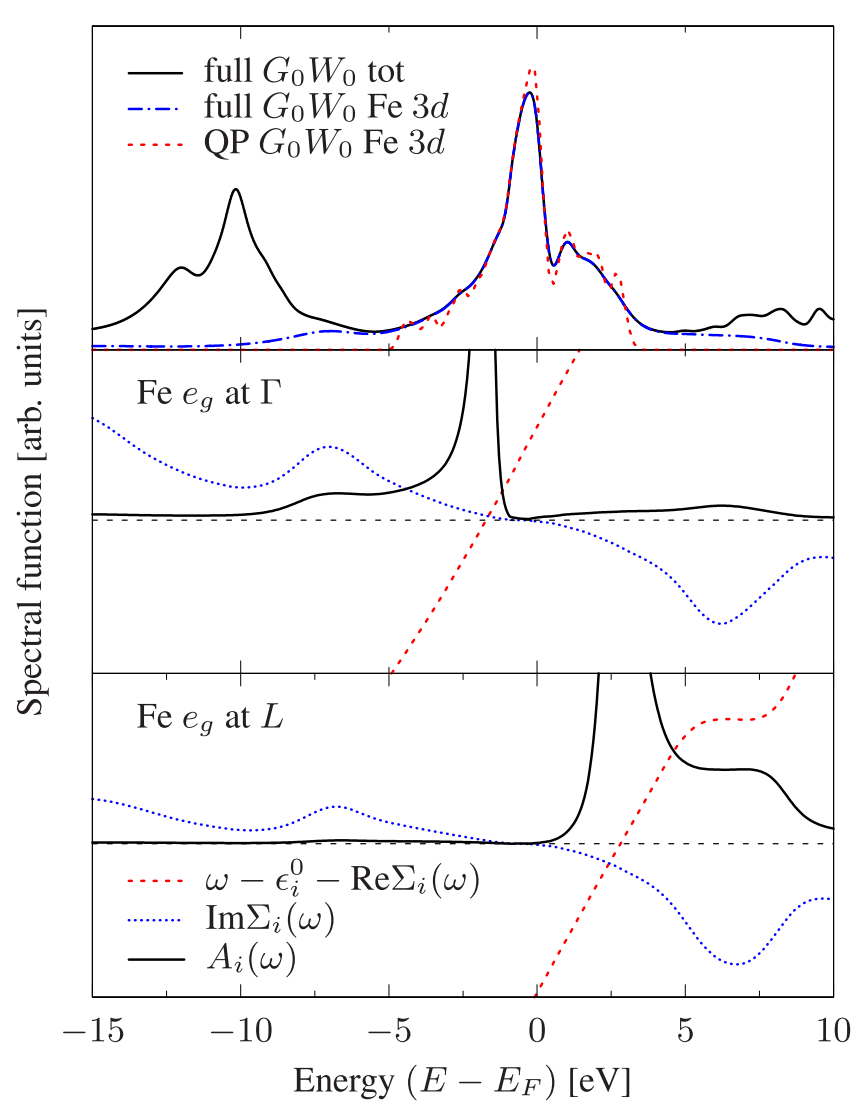

FIG. 6. Upper panel: Spectral function calculated with the full dynamical $G_{0} W_{0}$ (black solid line). For Fe $3 d$ states we report also the partial spectral function (blue dot-dashed line) and the QP spectral function (red dashed line). We notice that, for comparison, the QP spectrum has been corrected introducing the corresponding renormalization factor $Z_{i}$ which on average is 0.64 for the Fe $3 d$ bands. Middle and bottom panels: Spectral function $A_{i}(\omega)$ (solid black line), $\omega-\epsilon_{i}^{0}-\operatorname{Re} \Sigma_{i}(\omega)$ (red dashed line), and $\operatorname{Im} \Sigma_{i}(\omega)$ (blue dotted line) for the $\mathrm{Fe} e_{g}$ states at the $\Gamma$ and $L$ points. The quasiparticle energy $\epsilon_{i}^{\mathrm{QP}}$ is given by the solution of the equation $\omega-\epsilon_{i}^{0}-\operatorname{Re} \Sigma_{i}(\omega)=0$.

to $\delta_{i}^{R / A}(\omega)$. By analyzing the satellites of the $e_{g}$ states at the $\Gamma$ (for occupied $e_{g}$ states) and $L$ (for unoccupied $e_{g}$ states) points (see middle and lower panel of Fig. 6), we identify the same satellites as in the total spectral function in the upper panel of Fig. 6. A similar scenario occurs for the $t_{2 g}$ bands. This analysis is based on the expression of the total spectral function $A(\omega)=\sum_{i} A_{i}(\omega)$ with

$$
A_{i}(\omega)=\frac{1}{\pi} \frac{\left|\operatorname{Im} \Sigma_{i}(\omega)\right|}{\left|\omega-\epsilon_{i}^{0}-\operatorname{Re} \Sigma_{i}(\omega)\right|^{2}+\left|\operatorname{Im} \Sigma_{i}(\omega)\right|^{2}},
$$

where $\epsilon_{i}^{0}$ are the noninteracting single-particle energies, and $\operatorname{Im} \Sigma_{i}$ and $\operatorname{Re} \Sigma_{i}$ are the imaginary and real parts, respectively, of the self-energy. Both satellites are located in the vicinity of structures in the imaginary part of the $G W$ self-energy, which we checked to arise from a plasmon peak at $\approx 5 \mathrm{eV}$ in the energy-loss function.

\section{SUMMARY AND CONCLUSIONS}

We presented a detailed $a b$ initio study of the PES of paramagnetic $\mathrm{FeO}$ at ambient and high pressures using a refined version of the MEET. Our protocol is based on three important steps: (i) treating removal and addition parts of the PES separately; (ii) assuming the relative position of the MEET spectral features correct in both parts; and (iii) aligning the MEET energies corresponding to weakly correlated states with the LDA ones in order to get a rigid shift which decreases the band gap. We showed that this protocol gives an overall good description of the spectral function and of the spin configuration both at ambient and at high pressure even modeling the PM phase as nonmagnetic. This is a very important result of our method, contrary to state-of-the-art $a b$ initio methods which do not correctly describe the system at ambient pressure. However, more advanced approximations to the 1- and 2-RDMs are needed in order to make our method fully predictive. Work in this direction is in progress. For completeness we also report the results obtained using density-functional theory with the hybrid functional HSE06 and some variants of the $G W$ method (namely, scCOHSEX and $G_{0} W_{0}$ on top of LDA). Whereas the two methods are not appropriate at ambient pressure, increasing pressure they correctly describe the band-gap closing. The $G_{0} W_{0}$ photoemission spectrum of the metallic phase is in overall good agreement with DMFT results from literature. Besides the $d$-state quasiparticle peaks around the Fermi energy, two satellite peaks are also present, both stemming from plasmonic structures in the imaginary part of the $G_{0} W_{0}$ self-energy. These peaks are not described by the MEET within the current static approximation to the effective energy $\delta_{i}^{R / A}(\omega)$.

\section{ACKNOWLEDGMENTS}

This paper has been supported through Écoles Universitaires de Recherche (EUR) NanoX Grant No. ANR17-EURE-0009 in the framework of the "Programme des Investissements d'Avenir" and by Agence Nationale de la Recherche (ANR) Projects No. ANR-18-CE30-0025 and No. ANR-19-CE30-0011. The authors would like to thank Matteo Gatti and Claudia Rödl for fruitful discussions.
[1] V. R. Murthy, Early differentiation of the earth and the problem of mantle siderophile elements: A new approach, Science 253, 303 (1991).

[2] M. P. Pasternak, R. D. Taylor, R. Jeanloz, X. Li, J. H. Nguyen, and C. A. McCammon, High Pressure Collapse of Magnetism in $\mathrm{Fe}_{0.94} \mathrm{O}$ : Mössbauer Spectroscopy Beyond $100 \mathrm{GPa}$, Phys. Rev. Lett. 79, 5046 (1997).
[3] H. Ozawa, K. Hirose, K. Ohta, H. Ishii, N. Hiraoka, Y. Ohishi, and Y. Seto, Spin crossover, structural change, and metallization in NiAs-type $\mathrm{FeO}$ at high pressure, Phys. Rev. B 84, 134417 (2011).

[4] K. Ohta, R. E. Cohen, K. Hirose, K. Haule, K. Shimizu, and Y. Ohishi, Experimental and Theoretical Evidence for Pressure-Induced Metallization in $\mathrm{FeO}$ with 
Rocksalt-Type Structure, Phys. Rev. Lett. 108, 026403 (2012).

[5] T. Atou, M. Kawasaki, and S. Nakajima, Electronic transition of cobalt monoxide under high-pressure, Jpn. J. Appl. Phys. 43, L1281 (2004).

[6] J.-P. Rueff, A. Mattila, J. Badro, G. Vankó, and A. Shukla, Electronic properties of transition-metal oxides under high pressure revealed by X-ray emission spectroscopy, J. Phys.: Condens. Matter 17, S717 (2005).

[7] T. Yagi, T. Suzuki, and S.-I. Akimoto, Static compression of wüstite $\left(\mathrm{Fe}_{0.98} \mathrm{O}\right)$ to $120 \mathrm{GPa}$, J. Geophys. Res. Solid Earth 90, 8784 (1985).

[8] C. S. Yoo, B. Maddox, J.-H. P. Klepeis, V. Iota, W. Evans, A. McMahan, M. Y. Hu, P. Chow, M. Somayazulu, D. Häusermann, R. T. Scalettar, and W. E. Pickett, First-Order Isostructural Mott Transition in Highly Compressed Mno, Phys. Rev. Lett. 94, 115502 (2005).

[9] A. G. Gavriliuk, I. A. Trojan, and V. V. Struzhkin, InsulatorMetal Transition in Highly Compressed NiO, Phys. Rev. Lett. 109, 086402 (2012).

[10] D. G. Isaak, R. E. Cohen, M. J. Mehl, and D. J. Singh, Phase stability of wüstite at high pressure from first-principles linearized augmented plane-wave calculations, Phys. Rev. B 47, 7720 (1993).

[11] R. E. Cohen, I. I. Mazin, and D. G. Isaak, Magnetic collapse in transition metal oxides at high pressure: Implications for the earth, Science 275, 654 (1997).

[12] A. O. Shorikov, Z. V. Pchelkina, V. I. Anisimov, S. L. Skornyakov, and M. A. Korotin, Orbital-selective pressuredriven metal to insulator transition in $\mathrm{FeO}$ from dynamical mean-field theory, Phys. Rev. B 82, 195101 (2010).

[13] I. Leonov, Metal-insulator transition and local-moment collapse in FeO under pressure, Phys. Rev. B 92, 085142 (2015).

[14] I. Leonov, L. Pourovskii, A. Georges, and I. A. Abrikosov, Magnetic collapse and the behavior of transition metal oxides at high pressure, Phys. Rev. B 94, 155135 (2016).

[15] G. Trimarchi, Z. Wang, and A. Zunger, Polymorphous band structure model of gapping in the antiferromagnetic and paramagnetic phases of the mott insulators $\mathrm{MnO}, \mathrm{FeO}, \mathrm{CoO}$, and NiO, Phys. Rev. B 97, 035107 (2018).

[16] S. Di Sabatino, J. A. Berger, and P. Romaniello, Many-body effective energy theory: Photoemission at strong correlation, J. Chem. Theory Comput. 15, 5080 (2019).

[17] A. V. Krukau, O. A. Vydrov, A. F. Izmaylov, and G. E. Scuseria, Influence of the exchange screening parameter on the performance of screened hybrid functionals, J. Chem. Phys. 125, 224106 (2006).

[18] T. L. Gilbert, Hohenberg-Kohn theorem for nonlocal external potentials, Phys. Rev. B 12, 2111 (1975).

[19] S. Sharma, J. K. Dewhurst, N. N. Lathiotakis, and E. K. U. Gross, Reduced density matrix functional for many-electron systems, Phys. Rev. B 78, 201103(R) (2008).

[20] N. N. Lathiotakis, S. Sharma, J. K. Dewhurst, F. G. Eich, M. A. L. Marques, and E. K. U. Gross, Density-matrix-power functional: Performance for finite systems and the homogeneous electron gas, Phys. Rev. A 79, 040501(R) (2009).

[21] S. Sharma, J. K. Dewhurst, S. Shallcross, and E. K. U. Gross, Spectral Density and Metal-Insulator Phase Transition in Mott Insulators within Reduced Density Matrix Functional Theory, Phys. Rev. Lett. 110, 116403 (2013).
[22] N. N. Lathiotakis, N. Helbig, and E. K. U. Gross, Performance of one-body reduced density-matrix functionals for the homogeneous electron gas, Phys. Rev. B 75, 195120 (2007).

[23] S. Di Sabatino, J. A. Berger, L. Reining, and P. Romaniello, Photoemission spectra from reduced density matrices: The band gap in strongly correlated systems, Phys. Rev. B 94, 155141 (2016).

[24] R. M. Martin, L. Reining, and D. M. Ceperley, Interacting Electrons: Theory and Computational Approaches (Cambridge University, Cambridge, England, 2016).

[25] R. Zimmermann, P. Steiner, R. Claessen, F. Reinert, S. Hüfner, P. Blaha, and P. Dufek, Electronic structure of 3d-transitionmetal oxides: On-site coulomb repulsion versus covalency, J. Phys.: Condens. Matter 11, 1657 (1999).

[26] Computer code ELK, http://elk.sourceforge.net/.

[27] G. Kresse and J. Furthmüller, Efficient iterative schemes for $a b$ initio total-energy calculations using a plane-wave basis set, Phys. Rev. B 54, 11169 (1996).

[28] M. Shishkin and G. Kresse, Implementation and performance of the frequency-dependent $G W$ method within the paw framework, Phys. Rev. B 74, 035101 (2006).

[29] M. Shishkin, M. Marsman, and G. Kresse, Accurate Quasiparticle Spectra from Self-Consistent $G W$ Calculations with Vertex Corrections, Phys. Rev. Lett. 99, 246403 (2007).

[30] J. Paier, M. Marsman, K. Hummer, G. Kresse, I. C. Gerber, and J. G. Ángyán, Screened hybrid density functionals applied to solids, J. Chem. Phys. 124, 154709 (2006).

[31] P. E. Blöchl, Projector augmented-wave method, Phys. Rev. B 50, 17953 (1994).

[32] G. Kresse and D. Joubert, From ultrasoft pseudopotentials to the projector augmented-wave method, Phys. Rev. B 59, 1758 (1999).

[33] X. Gonze, G.-M. Rignanese, M. Verstraete, J.-M. Beuken, Y. Pouillon, R. Caracas, F. Jollet, M. Torrent, G. Zerah, M. Mikami et al., A brief introduction to the ABINIT software package, Z. Kristallogr. 220, 558 (2005)

[34] X. Gonze, B. Amadon, P.-M. Anglade, J.-M. Beuken, F. Bottin, P. Boulanger, F. Bruneval, D. Caliste, R. Caracas, M. Côté, T. Deutsch, L. Genovese, Ph. Ghosez, M. Giantomassi, S. Goedecker, D. R. Hamann, P. Hermet, F. Jollet, G. Jomard, S. Leroux, M. Mancini, S. Mazevet, M. J. T. Oliveira, G. Onida, Y. Pouillon, T. Rangel, G.-M. Rignanese, D. Sangalli, R. Shaltaf, M. Torrent, M. J. Verstraete, G. Zerah, and J. W. Zwanziger, ABINIT: First-principles approach to material and nanosystem properties, Comput. Phys. Commun. 180, 2582 (2009).

[35] B. Hentschel, Stoichiometric $\mathrm{FeO}$ as metastable intermediate of the decomposition of wustite at $225^{\circ} \mathrm{C}, \mathrm{Z}$. Naturforsch. Teil A 25, 1996 (1970).

[36] D. R. Hamann, Optimized norm-conserving vanderbilt pseudopotentials, Phys. Rev. B 88, 085117 (2013).

[37] M. J. van Setten, M. Giantomassi, E. Bousquet, M. J. Verstraete, D. R. Hamann, X. Gonze, and G.-M. Rignanese, The PseudoDojo: Training and grading a 85 element optimized norm-conserving pseudopotential table, Comput. Phys. Commun. 226, 39 (2018).

[38] S. Lebègue, B. Arnaud, M. Alouani, and P. E. Bloechl, Implementation of an all-electron GW approximation based on the projector augmented wave method without plasmon pole approximation: Application to $\mathrm{Si}, \mathrm{SiC}, \mathrm{AlAs}, \mathrm{InAs}, \mathrm{NaH}$, and KH, Phys. Rev. B 67, 155208 (2003). 
[39] We notice that the equilibrium volume used in Ref. [13] is the result of a geometry optimization within DMFT, whereas we use the experimental value. Therefore in order to compare our results with the results of Ref. [13] we use an interpolation of the data reported in Ref. [13].

[40] H. K. Bowen, D. Adler, and B. H. Auker, Electrical and optical properties of $\mathrm{FeO}$, J. Solid State Chem. 12, 355 (1975).

[41] C. Rödl, F. Fuchs, J. Furthmüller, and F. Bechstedt, Quasiparticle band structures of the antiferromagnetic transition-metal oxides $\mathrm{MnO}, \mathrm{FeO}, \mathrm{CoO}$, and $\mathrm{NiO}$, Phys. Rev. B 79, 235114 (2009).
[42] S. V. Faleev, M. van Schilfgaarde, and T. Kotani, All-Electron Self-Consistent $G W$ Approximation: Application to $\mathrm{Si}, \mathrm{MnO}$, and NiO, Phys. Rev. Lett. 93, 126406 (2004).

[43] S. Das, J. E. Coulter, and E. Manousakis, Convergence of quasiparticle self-consistent $G W$ calculations of transition-metal monoxides, Phys. Rev. B 91, 115105 (2015).

[44] L. Hedin, New method for calculating the one-particle Green's function with application to the electron-gas problem, Phys. Rev. 139, A796 (1965).

[45] M. Gatti and M. Guzzo, Dynamical screening in correlated metals: Spectral properties of $\mathrm{SrV}_{3}$ in the $G W$ approximation and beyond, Phys. Rev. B 87, 155147 (2013). 